\title{
Fecal microbiota and diet compositions of muskox female adults and calves
}

\author{
Ji-Yeon Cheon ${ }^{1}$, Hyunjoon Cho ${ }^{1}$, Mincheol Kim² ${ }^{2}$,Hyun Je Park ${ }^{3}$, Tae-Yoon Park ${ }^{1}$, and \\ Won Young Lee ${ }^{1}$ \\ ${ }^{1}$ Korea Polar Research Institute \\ ${ }^{2}$ Korea Polar Res Inst KOPRI \\ ${ }^{3}$ Gangneung-Wonju National University
}

January 9,2022

\begin{abstract}
Gut microbiome is vertically transmitted by maternal lactation at birth in mammals. In this study, we investigated the gut microbiome and diet compositions of muskox, a large herbivore in the high Arctic. From muskox feces in Ella Island, East Greenland, we compared the microbiota composition using bacterial 16S rRNA gene sequencing and the dietary compositions of six female adults and four calves have been compared. Firmicutes was the most abundant bacterial phylum in both adults and calves, comprising $94.36 \%$ and $94.03 \%$, respectively. There were significant differences in the relative abundance of two Firmicutes families: the adults were mainly dominated by Ruminococcaceae $(73.90 \%)$, while the calves were dominated by both Ruminococcaceae (56.25\%) and Lachnospiraceae (24.00\%). Stable isotope analysis on the feces and eight referential plant samples in the study area showed that both adults and calves had similar ranges of $13 \mathrm{C}$ and $15 \mathrm{~N}$, possibly derived from the dominant diet plants of Empetrum nigrum and Salix glauca. Despite the similar diets, the different gut microbiome compositions in muskox adults and calves indicate that the gut microbiome of the calves may not be fully colonized yet as much as the one of the adults.
\end{abstract}

\section{Introduction}

Animals are associated with a diverse microbiome in their gut, which affects the host's health, immunity, and metabolites of the host (Kinross et al., 2011). The composition of the gut microbiome may change with host development, diets, and surrounding environments of the host (Eckburg et al., 2005; Xu \& Knight., 2015). Thus, gut microbiome may provide an important insight into ecology of host animals and be related to pathogen which can cause the zoonotic diseases (Andersen-Ranberg et al., 2018). However, to date, the gut microbiome researches have been primarily focused on human or captive animals, but gut microbiome and its related functions of wild animals remain poorly understood (Davidson et al., 2020).

In mammals, gut microbiome can be vertically transmitted since birth though parental care during lactation phase by direct delivery of maternal materials to offspring, so that it has a significant impact on the gut microbiome formation in early growth stage of the offspring (Chu et al., 2016; Wang et al., 2020). In a mouse model study, it has been reported that most microbiota genera had been vertically transmitted over generations (Moeller et al. 2018). In addition to the vertical transmission, diet is also a major factor to facilitate the gut microbiome formation. Microbiota can be also indirectly affected by diet acquisitions of new-borns in different food conditions provided by nursing mothers (Frese et al. 2015). Since the composition of a starter diet can vary among families, host diet can shape microbial structure and functions for digestion.

In this study, we investigated the feces of a wild herbivore from female adults and calves in the high Arctic environments, collected during the summer. The muskox (Ovibos moschatus) is a large herbivore mammal 
that inhabits in the Arctic environments (Salgado-Flores et al., 2016). The dominant diets are willows (Salix spp.) in summer, graminoids (Carex , Eriophorum ) in winter (Gustine et al., 2014; Thing et al., 1987). Although the breeding season varies depending on the annual temperature (Schmidt et al., 2020), calves are usually born in April to May and start grazing from one week after birth, being closely attached to their mothers. Calves completely wean after one year (Adamczewski et al., 1994). By collecting fresh feces, the gut microbiomes and diet compositions were compared between the female adults and calves using bacterial $16 \mathrm{~S}$ rRNA gene sequencing and stable isotopes analysis. Here we questioned 1) if muskoxen have different gut microbiomes with ages (female adults vs. calves) and 2) if the two age groups have similar diets.

\section{Materials and methods}

\section{Study site and fecal sample collection}

The samples for this study were collected from Ella Island ( $72^{\circ} 50^{\prime} \mathrm{N}, 25^{\circ} 00^{\prime} \mathrm{W}$; Figure $\left.1 \mathrm{~A}\right)$, which is located in East Greenland, during August in 2019. Ella Island presents a dry environment with low temperatures not exceeding 10 even during summer (Kottek et al., 2006). In Willow, grasses, sedges, especially dwarf shrubs are dominant vegetation (Arndal et al., 2009).
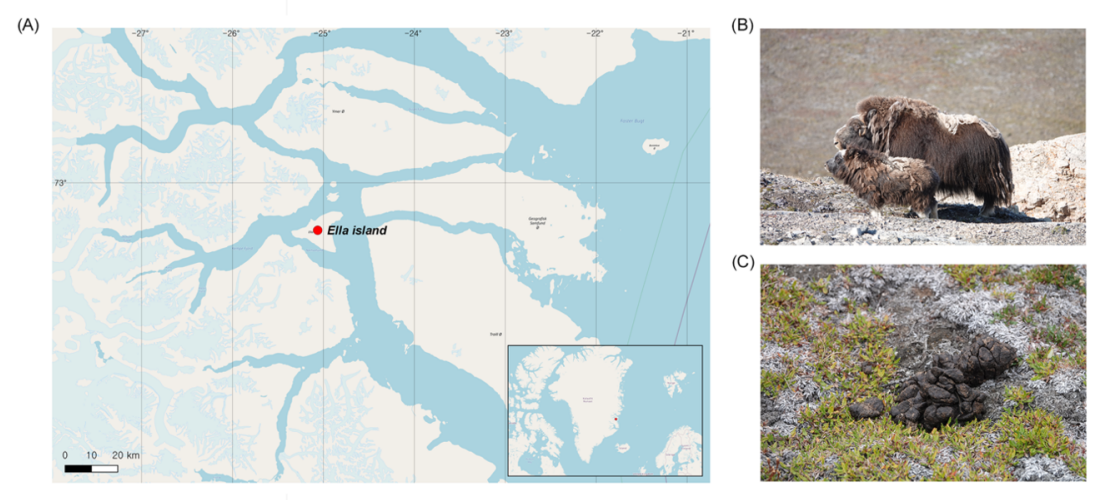

Figure 1. (A) The location of our study site (Ella Island in East Greenland, at latitude $\mathbf{7 2}^{\circ}$ 50' $\mathrm{N}$ and longitude 25 00' W). (B) Muskox female adult and calf in August 2019. (C) An example of muskox fecal sample.

Ten muskox fecal samples were collected; six from muskox female adults and four from calves (four pairs of mother and calf and two females with no calf, Figure 1B; muskox feces, Figure C). Among them, eight samples comprise four pairs of mother and calf. All samples had been kept in Ethanol solution (99\%) until the DNAs were extracted. We also sampled leaves, stems, and fruits of 8 plant species as candidate prey sources from the ground where the muskox foraged.

\section{DNA extraction, amplification and sequencing}

Fecal DNA was individually extracted from sub-samples at more than 3 different regions of the original sample, using the QIAGEN QIAamp fast stool mini kit as per the manufacturer's instructions. DNA was amplified targeting the V3-V4 region of bacterial $16 \mathrm{~S}$ rRNA gene using primers, 341F (5'CCTAGGGGNGGCWGCAG-3') and 805R (5'GACTACHVGGGTATCTAATCC-3') (Klindworth et al., 2013), and amplification was done using the following protocol: one denaturation step of $94^{\circ} \mathrm{C}$ for 3 min; 5 cycles of denaturation at $94^{\circ} \mathrm{C}$ for $15 \mathrm{~s}$ and extension at $65^{\circ} \mathrm{C}$ for $60 \mathrm{~s}, 20$ cycles of denaturation at $94^{\circ} \mathrm{C}$ for $1 \mathrm{~min}$, annealing at $55^{\circ} \mathrm{C}$ for $20 \mathrm{~s}$ and extension at $72^{\circ} \mathrm{C}$ for $30 \mathrm{~s}$, and a final extension at $72^{\circ} \mathrm{C}$ for 5 min. Sequencing library construction and amplicon sequencing were performed at Macrogen (Seoul, South Korea) using a $2 \times 300$ bp Illumina MiSeq sequencing system (Illumina, USA).

Bioinformatic analyses 
The adapter and primers from the raw sequence reads were trimmed using Cutadapt v2.10 (Martin et al., 2011). Bioinformatics pipeline was run using DADA2 v1.16 (Callahan et al., 2016) to infer amplicon sequence variants (ASVs) with single-nucleotide resolution. For quality trimming, a more relaxed filtering option was applied on the reverse reads as maxEE $=\mathrm{c}(2,5)$, and the low-quality sequence tails were removed from the forward and reverse reads with truncLen $=\mathrm{c}(270,210)$. Bacterial taxonomy was assigned to representative ASV sequences using DADA2 implementation of RDP naïve Bayesian classifier based on the EzBiocloud database (Yoon et al., 2017). Sequences matched to the Eukaryota, Archaea or Cyanobacteria were removed from the dataset. Sequences are available in the NCBI Sequence Read Archive (SRA) database under accession number PRJNA753257.

\section{Stable isotopes analysis}

For stable isotope analysis, 1mg muskox feces and 1.0-1.5mg plant tissue samples (extracted evenly from leaves, stems, and fruits if available) were homogenized. Each sample was freeze-dried and then prepared with a stable isotope ratio mass spectrometer system (IsoPrime 100; Cheadle, UK) with an elemental analyser (vario MICRO cube; Elementar, Hanau, Germany). Purified $\mathrm{CO}_{2}$ and $\mathrm{N}_{2}$ were used as the sample analysis gas and the isotopic reference gases. GC column resolve $\mathrm{CO}_{2}$ from $\mathrm{N}_{2}$ and reduction column filled with copper wires reduce $\mathrm{N}_{2}$. All results are reported with delta notation, in parts per thousand (repeated six times of this analysis.

$\delta^{13} \mathrm{C}$ and $\delta^{15} \mathrm{~N}=\left(\mathrm{R}_{\text {sample }} / \mathrm{R}_{\text {standard }}-1\right) * 1,000($

The international reference materials of sucrose (ANU C12H22O11; NIST, Gaithersburg, MD, USA) for $\delta 13 \mathrm{C}$ and ammonium sulfate ([NH4]2SO4; NIST) for $\delta 15 \mathrm{~N}$ were analyzed for calibration of reference gases and the internal standard (acetanilide; Thermo Scientific). The analytical precision was based on 10 replicate measurements of acetanilide and was within 0.12

We used simmr Bayesian mixing model in the $\mathrm{R}$ with five plant food sources, which were known as muskox diet (Forchhammer \& Boomsma., 1995; Gustine et al., 2014; Mosbacher et al., 2016), to estimate dietary fiber composition in muskox diets (stable isotopes mixing models in R; Parnell., 2019). The detailed procedure for running the simmr mixing model is described in Swan et al. (2020).

\section{Statistical analysis}

To correct the differences in the number of reads, all samples were subsampled to the level of the smallest number of reads found in the samples. Bray-Curtis dissimilarities between all sample pairs were calculated using a Hellinger-transformed ASVs abundance matrix, and visualized using non-metric multidimensional scaling (NMDS). A nonparametric multivariate test (Permutational Multivariate Analysis of Variance, which is called 'PERMANOVA') was used to test for differences in bacterial community structure between the two groups of the muskox using PRIMER 6 and PERMANOVA+ (Clarke \& Tobutt., 2003).

Age group (fixed with two levels; adult and calf) was considered as a fixed component, and p-values were obtained using 999 permutations. We used the 3 indices to estimate the bacterial diversity and compared the diversity values between two groups of the muskox by age with $t$ test and Fligner-Killeen test. Rarefaction curve, stable isotopes analysis results were generated using $\mathrm{R}$ packages (version 4.0.5, http://www.Rproject.org).

Bacterial functional abundances were inferred using PICRUSt2 v.2.3.0b (Douglas et al., 2020), and the predicted microbial functions (KEGG orthologs) were visualized with Principal Coordinates Analysis (PCoA) plot.

\section{Results}

We obtained a total of 335,970 high-quality bacterial 16S rRNA gene sequences from all muskox fecal samples, ranging from 19,222 to 42,610 sequences per sample. The rarefaction curves displayed that it almost attained the saturation plateau showing that the sample coverages were large enough to estimate the ASV richness (Figure 2). 


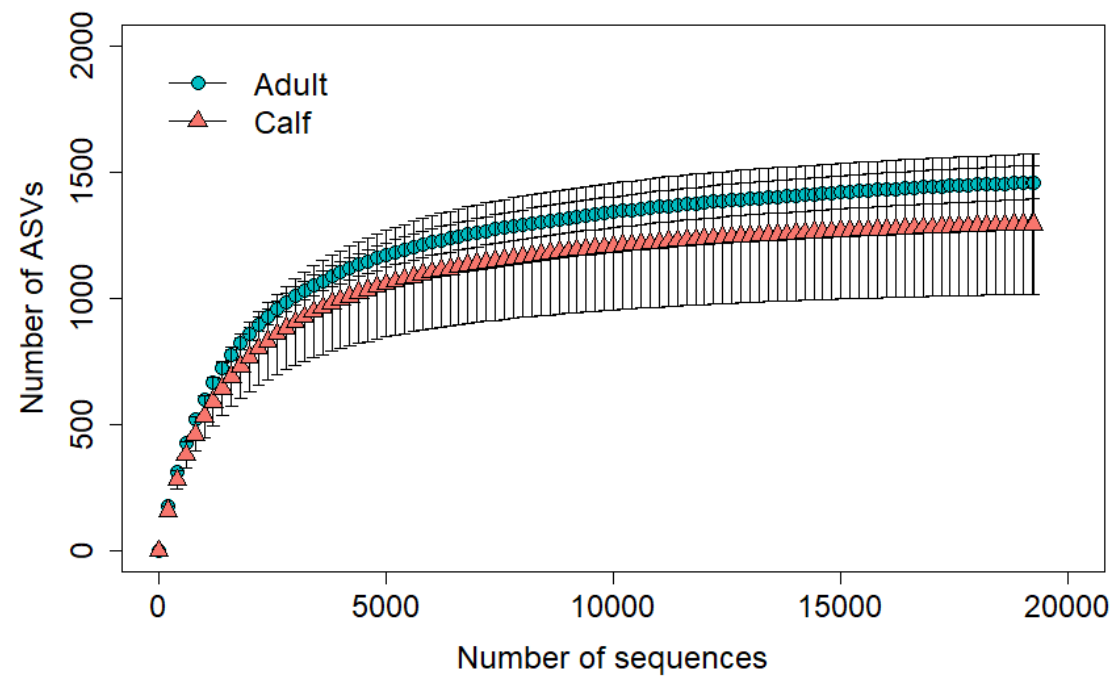

Figure 2. Rarefaction curves of fecal bacterial communities between muskox adult and calf groups

Adult bacterial diversity was $14.47 \%, 5.60 \%, 30.15 \%$ higher than that of calves in Chao, Shannon, and Invsimpson indices, respectively (Chao index; adults $=1543.9$, calves $=1348.7$, Shannon index; adults $=6.86$, calves $=6.48$, Invsimpson; adults $=752$, calves $=577.8$ on average), but the differences were not statistically significant (t-tests; Chao, $\mathrm{p}=0.56$; Shannon, $\mathrm{p}=0.450$; Invsimpson, $\mathrm{p}=0.430$; Figure 3 ). Instead, there were differences in group variances of three diversity indices between adults and calves (Fligner-Killeen tests; Chao, $\mathrm{p}=0.029$; Shannon, $\mathrm{p}=0.0013$; Invsimpson, $\mathrm{p}=0.019$; Figure 3).

The NMDS plot showed bacterial community differences between muskox adults and calves (PERMANOVA, pseudo-F $=1.69, \mathrm{p}=0.003$; Figure 4 ). 

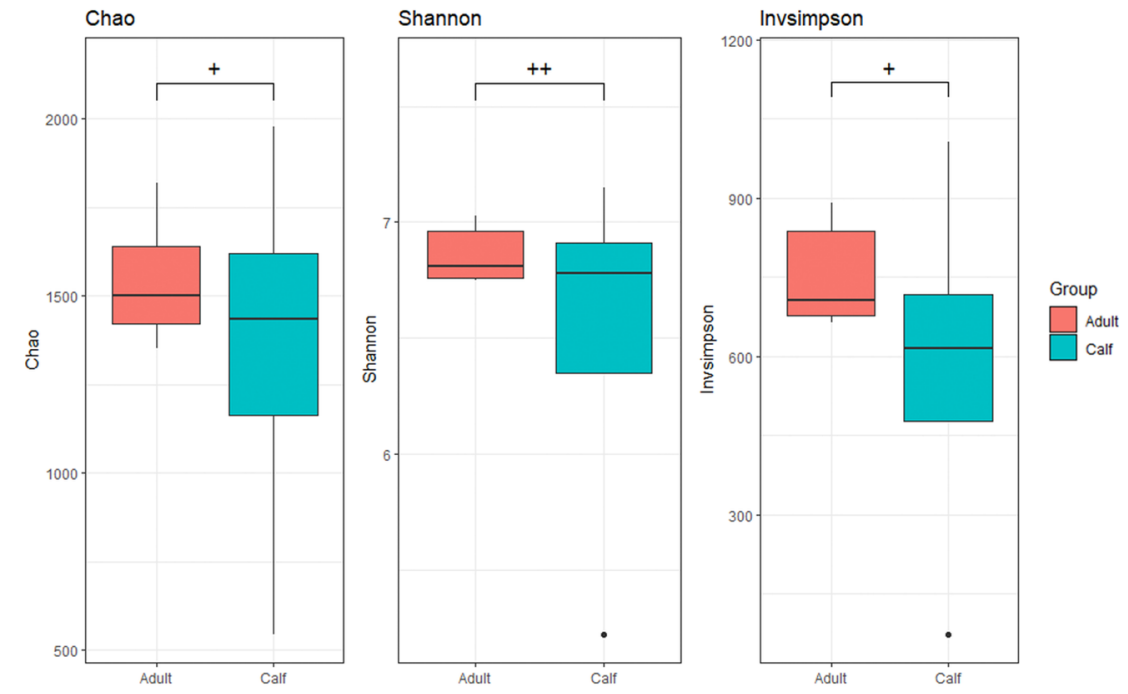

Figure 3. Bacterial alpha-diversity (3 indices; Chao, Shannon and Invsimpson) in muskox adults $(n=6)$ and calves $(n=4)$ presented on box-whisker plots. No significant mean differences were detected between adults and calves (t-tests; Chao, $\mathrm{p}=0.56$; Shannon, $\mathrm{p}=0.45$; Invsimpson, $p=0.43$ ) but homogeneity of variance Fligner-Killeen test; ("+" means $p<0.05$, " $++"$ means $\mathrm{p}<0.01$; Chao, $\mathrm{p}=0.03$; Shannon, $\mathrm{p}=0.0013$; Invsimpson, $\mathrm{p}=0.02)$.

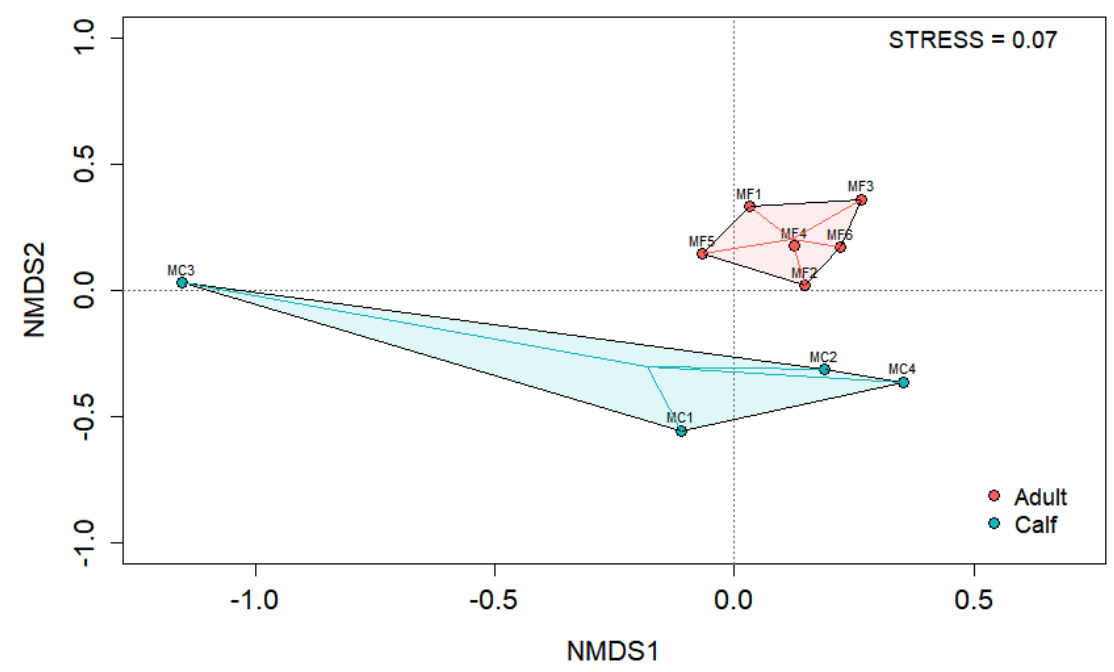

Figure 4. Non-metric multidimensional scaling (NMDS) plot of muskox fecal bacterial communities using Bray-Curtis dissimilarity measures. All the points within each group were connected to the group centroid. 
(A)

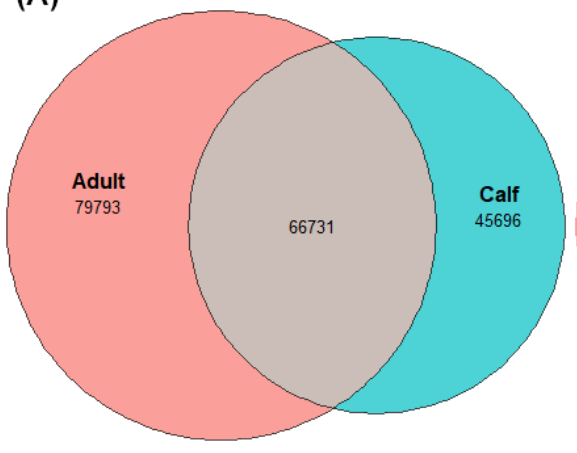

(B)

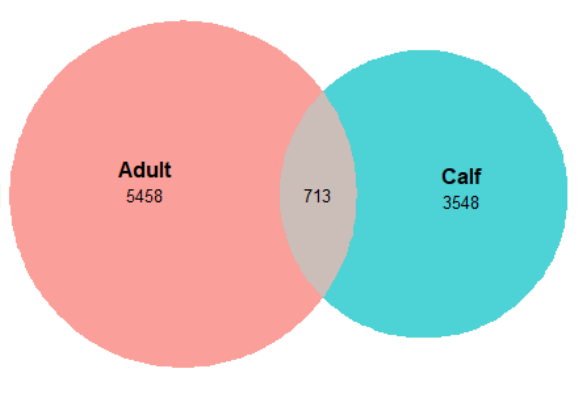

Figure 5. Venn diagram displaying (A) the total sequence reads (B) the number of unique and shared bacterial ASVs in adult and calf groups

We attempted to identify bacterial ASVs which were shared between different groups of the muskox. $34.72 \%$ (66731/125489) of the total sequence reads were shared, $7.33 \%(713 / 9719)$ of the total ASVs were shared between adults and calves (Figure 5). In the shared ASVs, Ruminococcaceae (adults: $77.96 \%$, calves: $60.64 \%$ ) and Lachnospiraceae (adults: $8.79 \%$, calves: $26.69 \%$ ) were abundant. 

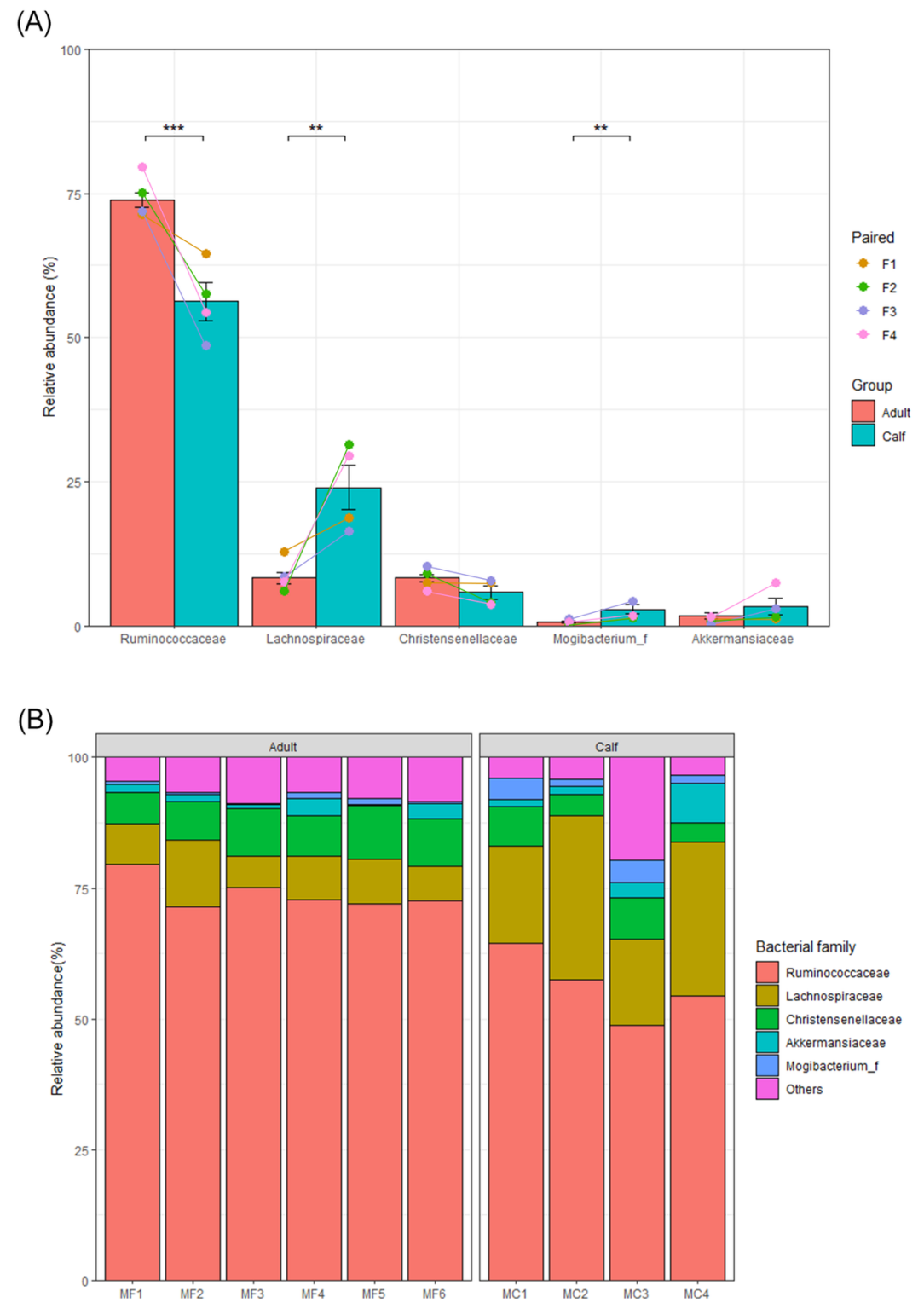

Figure 6. (A) Relative abundance of dominant bacteria family of the total number of ASVs in muskox adults and calves. Bar plot showed the relative abundance of adults $(n=6)$ and calves $(n=4)$, t-tests; Asterisks indicate the significance of the statistical test of differences between adults and calves (Asterisks $*$ means $\mathrm{p}<0.05$, ** means $\mathrm{p}<0.01$, *** means $\mathrm{p}<$ 0.001; Ruminococcaceae, $\mathrm{p}=\mathbf{0 . 0 0 0 4}$; Lachnospiraceae, $\mathrm{p}=0.0012$; Christensenellaceae, $\mathrm{p}=$ 0.070; Mogibacterium_f, $\mathrm{p}=0.009$; Akkermansiaceae, $\mathrm{p}=0.270$ ). Points show the relative abundance of adults $(n=4)$ and calves $(n=4)$ with paired samples. (B) Distribution of bacterial families across all fecal samples from muskox individuals.

At the phylum level, the muskox gut microbiome was dominated by Firmicutes (on average 94.36\%, 94.03\%) and Verrucomicrobia $(1.77 \%, 3.31 \%)$ respectively in both adults and calves. These two phyla accounted 
for $91.16 \%$ of the total sequences from all the samples. At the family level, we found that five families were dominant: Ruminococcaceae $(73.90 \%, 56.25 \%)$, Lachnospiraceae $(8.27 \%, 24.00 \%)$, Christensenellaceae $(8.28 \%, 5.76 \%)$, Mogibacterium_f $(0.65 \%, 2.86 \%)$, and Akkermansiaceae $(1.72 \%, 3.30 \%)$ (Figure 6AB). Three microbial families were significantly different in their relative abundances between muskox adults and calves (Ruminococcaceae, $\mathrm{p}=0.0004$; Lachnospiraceae, $\mathrm{p}=0.001$; Mogibacterium_f, $\mathrm{p}=0.009$; t-tests; Figure $6 \mathrm{~A}$ ). Although the four pairs of adult and calves did not show significances (paired t-tests; indicated by colored lines in Figure 6A), Ruminococcaceae and Christensenellaceae showed consistent increases and Lachnospiraceae, Mogibacterium_f and Akkermansiaceae showed consistent decreases in all pairs.

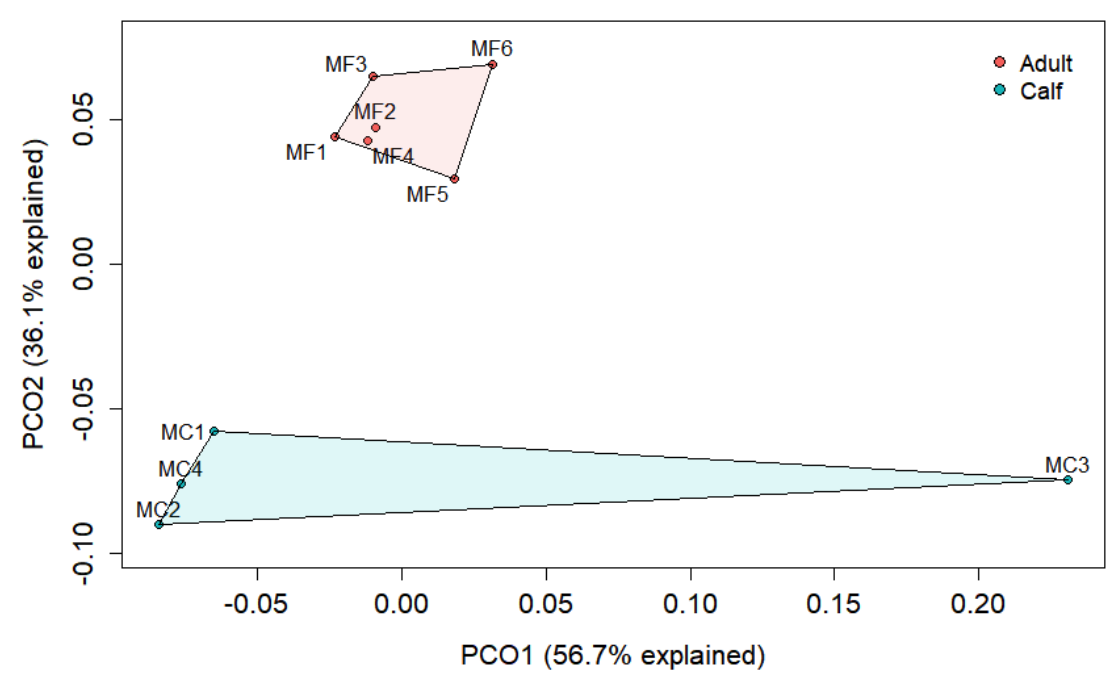

Figure 7. Principal Coordinates Analysis (PCoA) plot of PICRUSt2-predicted functions of muskox fecal microbiota using Bray-Curtis dissimilarity measures (adults: MF1-6, n=6), calves: $\mathrm{MC1}-4, \mathrm{n}=4)$.

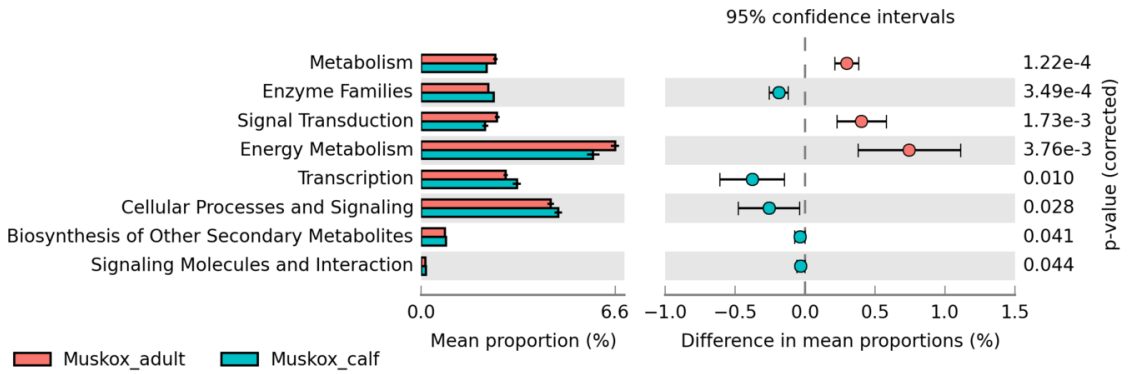

Figures 8. The PICRUSt2-predicted microbial functions with significant differences between the muskox adults and calves at level 2 KEGG functional categories.

Microbial functional structure differed significantly between muskox adults and calves (PERMANOVA, pseudo-F $=3.63, \mathrm{p}=0.012$ ) (Figure 7). Among predicted functions at KEGG level 2, 8 functions were significantly different between muskox adults and calves (Figure 8). Energy metabolism pathway was the dominant pathway in both muskox adults and calves $(6.63 \%$ vs. $5.88 \%, \mathrm{p}=0.004)$, but the adults had higher nitrogen metabolism functions than the calves $(0.77 \%$ vs. $0.65 \%, \mathrm{p}<0.001)$. Other metabolism pathways 
did not show significant differences between the two groups (carbohydrate metabolism, $0.11 \%$ and $0.13 \%, \mathrm{p}$ $=0.29$; fatty acid metabolism, $0.41 \%$ and $0.33 \% \mathrm{p}=0.49$; glyoxylate and dicarboxylate metabolism, $0.76 \%$ and $0.65 \%, \mathrm{p}=0.15$; butanoate metabolism, $0.98 \%$ and $0.84 \%, \mathrm{p}=0.33$; fatty acid biosynthesis, $0.60 \%$ and $0.57 \%, \mathrm{p}=0.21$; adults and calves, respectively; Figure 8).

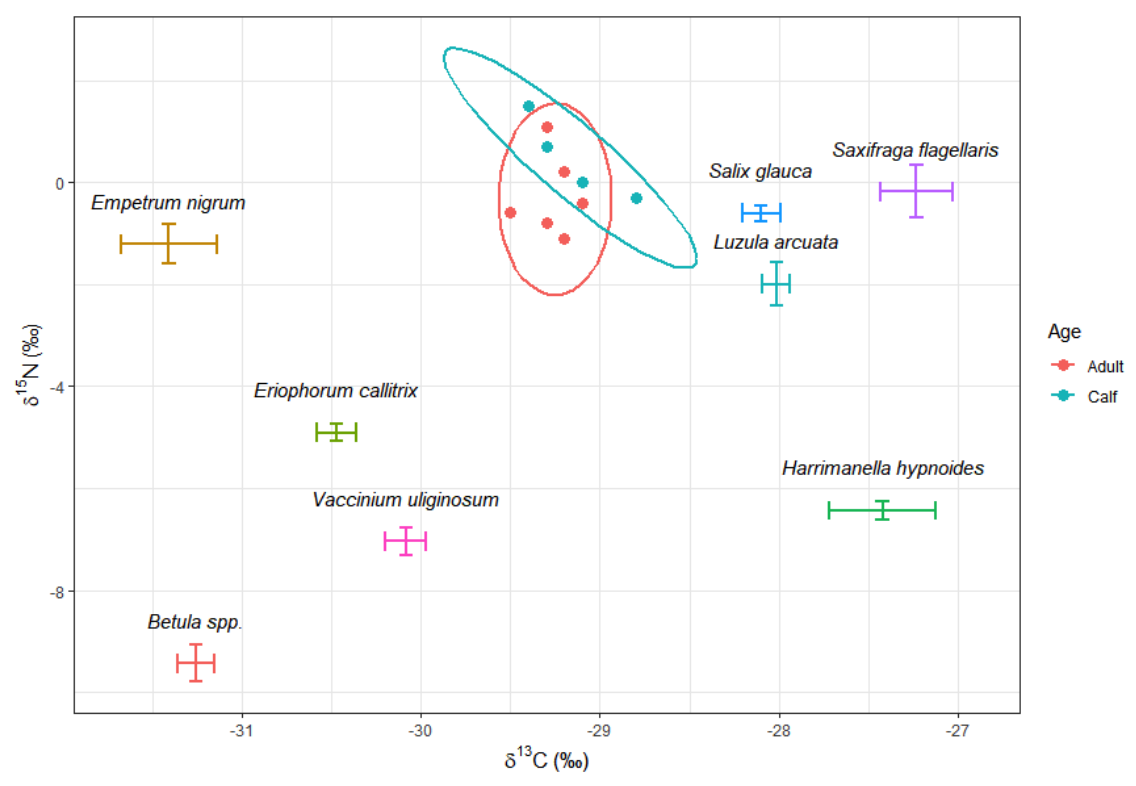

Figure 9. Stable isotopic niche for muskox adults $(\mathrm{n}=6)$, calves $(\mathrm{n}=4)$ and plants from study area $(\mathrm{n}=48$, Betula spp.,Empetrum nigrum, Eriophorum callitrix, Hamimanella hypnoides, Luzula arcuata, Salix glauca, Saxifraga flagellaris, Vaccinium uliginosum; six replicates in each species). Ellipses represent $90 \%$ confidence interval in muskox each group.E. nigrum (buddha gold), E. callitrix (dark green), L. arcuata (cyan), S. glauca (pure blue), and $S$. flagellaris (light violet) were previously noted as diets of muskox.

Stable isotopic niches for muskox adults $(n=6)$, calves $(n=4)$ and plants from study area ( 8 species) presented in Figure 9. Muskox adults and calves had similar values in $\delta^{13} \mathrm{C}$ and $\delta^{15} \mathrm{~N}$ (PERMANOVA, $\mathrm{p}=0.413$; adults, $\delta^{13} \mathrm{C}=-29.15 \pm 0.13, \delta^{15} \mathrm{~N}=-0.26 \pm 0.32$; calves, $\left.\delta^{13} \mathrm{C}=-29.15 \pm 0.13, \delta^{15} \mathrm{~N}=0.47 \pm 0.4\right)$. Two group ellipses were overlapped in $47.6 \%$ of area of adults and $48.1 \%$ of calves.

Each plant species had its distinctive value from others (Betulaspp., $\delta^{13} \mathrm{C}=-31.25 \pm 0.1, \delta^{15} \mathrm{~N}=-9.44 \pm$ 0.36; E. callitrix, $\delta^{13} \mathrm{C}=-30.47 \pm 0.1, \delta^{15} \mathrm{~N}=-4.9 \pm 0.18 ;$ E. nigrum, $\delta^{13} \mathrm{C}=-31.4 \pm 0.27, \delta^{15} \mathrm{~N}=-1.2$ $\pm 0.4 ;$ H. hypnoides, $\delta^{13} \mathrm{C}=-27.42 \pm 0.3, \delta^{15} \mathrm{~N}=-6.44 \pm 0.17 ;$ L. arcuata, $\delta^{13} \mathrm{C}=-28.02 \pm 0.07, \delta^{15} \mathrm{~N}=$ $-1.99 \pm 0.43 ;$ S. flagellaris, $\delta^{13} \mathrm{C}=-27.33 \pm 0.2, \delta^{15} \mathrm{~N}=-0.18 \pm 0.51 ;$ S. glauca, $\delta^{13} \mathrm{C}=-28.1 \pm 0.1, \delta^{15} \mathrm{~N}$ $=-0.6 \pm 0.15 ;$ V. uliginosum, $\left.\delta^{13} \mathrm{C}=-30.08 \pm 0.11, \delta^{15} \mathrm{~N}=-7.04 \pm 0.26\right)$. Among the 8 species, four plants (E. nigrum, L. arcuata, S. flagellaris and S. glauca) had similar $\delta^{15} \mathrm{~N}$ value (presenting a trophic level), with our muskoxen sample. 

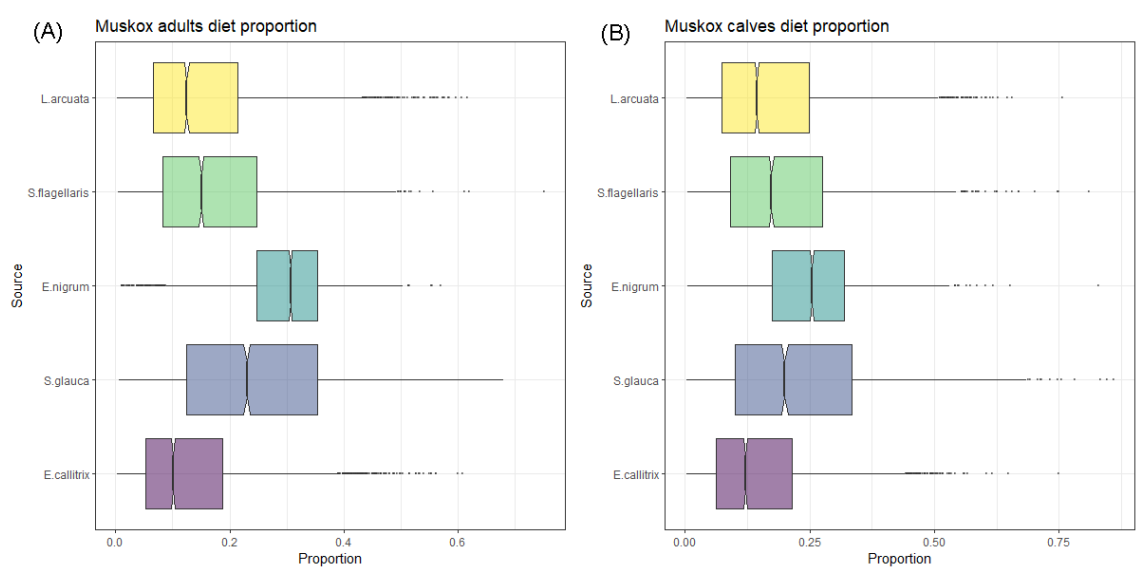

Figure 10. Stable isotopes mixing models of muskox diet proportion with five plants ( $L$. arcuata, S. flagellaris, E. nigrum, S. glauca, E. calltrix) presented on box-whisker plots in each muskox group, using simmr packages. (A) Proportions of five candidate plants of adults $($ L. arcuata $=0.15$, S. flagellaris $=0.17$, E. nigrum $=0.30$, S. glauca $=0.25$, E. callitrix $=$ 0.13) and (B) Proportions of five candidate plants of calves (L. arcuata $=0.17, S$. flagellaris $=0.20, E$. nigrum $=0.25$, S. glauca $=0.23, E$. callitrix $=0.15)$.

Stable isotope mixing models with five possible dietary plants represent the predicted proportions (Figure $10 \mathrm{AB}$ ). In both adults and calves, E. nigrum occupied the highest proportion of their diet (adults $=0.30$, calves $=0.25$ ), which was followed by $S$. glauca (adults $=0.25$, calves $=0.23$ ). Mixing models display that diet compositions were not different between the adults and calves (probabilistic Bayesian models; $L$. arcuata, $\mathrm{p}=0.54 ;$ S. flagellaris, $\mathrm{p}=0.54 ;$ E. nigrum, $\mathrm{p}=0.34 ;$ S. glauca, $\mathrm{p}=0.47 ;$ E. callitrix, $\mathrm{p}=0.58)$.

\section{Discussion}

Our results showed that the microbiome of muskox adults and calves have similar levels of alpha-diversity, at phylum and family level, although the calves showed higher variance values. The adults and calves had different bacterial communities even if the calves showed more diverse composition within the group compared to the adults. There was an apparent difference in the relative abundance of two Firmicutes families. The diet analysis indicates that the adults and calves had common dietary plant species. The different bacterial communities between the female adults and calves, which share similar diets, suggest that the gut microbiome in the calf group is still unstable and not fully colonized despite the dietary similarity. After birth, calves receive the gut microbiome from mothers and begin to form their own independent gut microbiome (Barko et al., 2018). Muskox calves graze from one week after birth, following mothers to choose dietary plants; it was also observed during our study period that the females and calves were foraging together. Diet analysis results also confirmed that the adults and calves had the common plants. Considering the breeding cycle (birth around March or April; Adamczewski et al., 1994), the calves in this study are assumed to be three or four months old. Thus, we predict that the calves may not finish constructing their gut microbiome by August.

The major dominant phylum of our fecal sample was Firmicutes. At the family level, Ruminococcaceae and Lachnospiraceae, which belong to the class Clostridia, phylum Firmicutes, were dominant to occupy more than $80 \%$ of total abundance. Ruminococcaceae and Lachnospiraceae were reported to encode carbohydrateactive enzymes for glycoside hydrolases and carbohydrate esterases in herbivores (Wang et al., 2016). Ruminococcaceae was also know to affect the secondary metabolite synthesis to get involved in host immunity, such as antibiotic biosynthesis (Gosalbes et al., 2011) by producing SCFAs (short chain fatty acids) for lipid metabolism and digestion (Morrison \& Preston., 2016) and by detoxifying the plant secondary metabolites (Kohl et al., 2014). Lachnospiraceae was reported to produce SCFAs for metabolism (Hao et al., 2017; Vacca 
et al., 2020), but also digest lactose by converting lactate into butyrate (Meehan \& Beiko., 2014). In our results, adults had more Ruminococcaceae and less Lachnospriaceae than calves. Such differences could be related to the microbial functions for host digestion and metabolism, depending on their need. We infer that the differences could result in the differential needs for digestion between the adults and calves since calves were still relying on the breast milk during the sampling period.

Predicted microbial functions indicates that energy metabolism was the most dominant (adults: 6.63\%, calves: $5.88 \%$ ). We found pathways to help digestion of dietary fibers for carbohydrates (carbohydrate metabolism, adults: $0.11 \%$, calves: $0.13 \%$ ) and lipids (fatty acid metabolism, adults: $0.41 \%$, calves: $0.33 \%$; glyoxylate and dicarboxylate metabolism, adults: $0.76 \%$, calves: $0.65 \%$; fatty acid biosynthesis, adults: $0.60 \%$, calves: $0.57 \%$ ).

Our microbial results correspond to the previous studies on large herbivores (Table 1). In the previous muskox studies, Firmicutes was the most dominant phylum (74-83\%) and Ruminococcaceae, Lachnospiraceae were the most dominant families (47-65\% and 13-16\%, respectively) (Salgado-Flores et al., 2016; Bird. 2019). In Svalbard and Norwegian reindeers, phylum Firmicutes and family Ruminococcaceae, Lachnospiraceae were also abundant (Zielińska et al., 2016; Sundset et al., 2007; Gruninger et al., 2014). From previous studies and our PICRUSt2 results, we estimate that Ruminococcaceae and Lachnospiraceae could promote cellulose metabolism for herbivores.

From the stable isotopes analysis, we found that adults and calves shared similar diet plants with E. nigrum and $S$. glaucabeing the dominant species. In previous studies, $S$. glauca were known to be a main food source of muskoxen, especially during the summer (Gustine et al., 2014; Thing et al., 1987), which shows a relatively high digestibility for muskoxen (Staaland \& Olesen 1992). E. nigrum has not been previously considered an impotant plant despite the potential vegetation records in a newly introduced area (in westeren Alaska, Ihl \& Klein., 2001). Our findings suggest that muskox adults and calves are dependent on the similar plant items and E. nigrumcan be additionally consumed by muskoxen at a specific time period in our study site. Considering the recent increase of E. nigrum with Arctic warming (Bråthen et al. 2018), it can become an important food sources for muskoxen.

In conclusion, our findings may provide ecological information for understanding the host and microbial interactions and shed light on the microbial functions for digestion in herbivores. In future studies, it will be interesting to analyze the detailed microbial functions in adults and calves related to the digestion and immune functions. Furthermore, it is necessary to record the diet compositions of the large herbivore animals and monitor their dietary changes since the Arctic ecosystem is rapidly changing with global warming.

\section{References}

Adamczewski, J. Z., Flood, P. F., Chaplin, R. K., \& Schaefer, J. A. (1994). Seasonal variation in intake and digestion of a high-roughage diet by muskoxen. Canadian Journal of Animal Science ,74 (2), 305-313. https://doi.org/10.4141/cjas94-042

Andersen-Ranberg, E. U., Barnes, C. J., Rasmussen, L., Salgado-Flores, A., Grøndahl, C., Mosbacher, J. B., Hansen, A. J., Sundset, M. A., Schmidt, N. M., \& Sonne, C. (2018). A comparative study on the faecal bacterial community and potential zoonotic bacteria of muskoxen (Ovibos moschatus) in Northeast Greenland, Northwest Greenland and Norway. Microorganisms , 6 (3), 76. https://doi.org/10.3390/microorganisms6030076

Arndal, M. F., Illeris, L., Michelsen, A., Albert K., Tamstorf, M., Hansen, B. U. (2009) Seasonal variation in gross ecosystem production, plant biomass, and carbon and nitrogen pools in five high Arctic vegetation types. Arctic, Antarctic, and Alpine Research ,41(2) , 164-173. https://doi.org/10.1657/1938-4246-41.2.164

Barko, P. C., McMichael, M. A., Swanson, K. S., \& Williams, D. A. (2018). The gastrointestinal microbiome: a review. Journal of Veterinary Internal Medicine, 32 (1), 9-25. https://doi.org/10.1111/jvim.14875

Bird, S., Prewer, E., Kutz, S., Leclerc, L.-M., Vilaca, S. T., \& Kyle, C. J. (2019). Geography, seasonality, and host-associated population structure influence the fecal microbiome of a genetically depauparate Arctic 
mammal. Ecology and Evolution , 9 (23), 13202-13217. https://doi.org/10.1002/ece3.5768

Bråthen, K. A., Gonzalez, V. T., \& Yoccoz, N. G. (2018). Gatekeepers to the effects of climate warming? Niche construction restricts plant community changes along a temperature gradient. Perspectives in Plant Ecology, Evolution and Systematics , 30 , 71-81. https://doi.org/10.1016/j.ppees.2017.06.005

Callahan, B. J., McMurdie, P. J., Rosen, M. J., Han, A. W., Johnson, A. J. A., \& Holmes, S. P. (2016). DADA2: high-resolution sample inference from Illumina amplicon data. Encyclopedia of Medical Immunology ,13 (7), 581-583. https://doi.org/10.1007/978-1-4614-9209-2_118-1

Chu, D. M., Meyer, K. M., Prince, A. L., \& Aagaard, K. M. (2016). Impact of maternal nutrition in pregnancy and lactation on offspring gut microbial composition and function. Gut Microbes , 7 (6), 459-470. https://doi.org/10.1080/19490976.2016.1241357

Clarke, J. B., \& Tobutt, K. R. (2003). Development and characterization of polymorphic microsatellites from Prunus avium 'Napoleon'.Molecular Ecology Notes , 3 (4), 578-580. https://doi.org/10.1046/j.14718286.2003.00517.x

Davidson, G. L., Raulo, A., \& Knowles, S. C. L. (2020) Identifying Microbiome-Mediated Behaviour in Wild Vertebrates. Trends in Ecology and Evolution, 35 (11), 972-980. https://doi.org/10.1016/j.tree.2020.06.014

Douglas, G. M., Maffei, V. J., Zaneveld, J. R., Yurgel, S. N., Brown, J. R., Taylor, C. M., Huttenhower, C., \& Langille Morgan G. I. (2020). PICRUSt2 for prediction of metagenome functions. Nature Biotechnology, 38 (6), 685-688. https://doi.org/10.1038/s41587-020-0550-z

Eckburg, P. B., Bik, E. M., Bernstein, C. N., Purdom, E., Dethlefsen, L., Sargent, M., Gill, S. R., Nelson, K. E., \& Relman, D. A. (2005). Diversity of the human intestinal microbial flora. Science ,308 , 1635-1638. https://doi.org/10.1126/science.1110591

Fadrosh, D. W., Ma, B., Gajer, P., Sengamalay, N., Ott, S., Brotman, R. M., \& Ravel, J. (2014). An improved dual-indexing approach for multiplexed 16S rRNA gene sequencing on the Illumina MiSeq platform.Microbiome , 2 (1), 1-7. https://doi.org/10.1186/2049-2618-2-6

Frese, S. A., Parker, K., Calvert, C. C., \& Mills, D. A. (2015) Diet shapes the gut microbiome of pigs during nursing and weaning. Microbiome , 3 , 28. https://doi.org/10.1186/s40168-015-0091-8

Gosalbes, M. J., Durbán, A., Pignatelli, M., Abellan, J. J., Jiménez-Hernández, N., Pérez-Cobas, A. E., Latorre, A., \& Moya, A. (2011). Metatranscriptomic approach to analyze the functional human gut microbiota. PLoS ONE , 6 (3), e17447. https://doi.org/10.1371/journal.pone.0017447

Gustine, D. D., Barboza, P. S., Addison, J., Shively, R., \& Oliver, L. (2014). Isotopic nitrogen in fecal fiber as an indicator of winter diet in caribou and muskoxen. Rapid Communications in Mass Spectrometry, 28 (6), 625-634. https://doi.org/10.1002/rcm.6825

Hao, Y. T., Wu, S. G., Xiong, F., Tran, N. T., Jakovlić, I., Zou, H., Li, W. X., \& Wang, G. T. (2017). Succession and fermentation products of grass carp (Ctenopharyngodon idellus ) hindgut microbiota in response to an extreme dietary shift. Frontiers in Microbiology ,8 , 1585. https://doi.org/10.3389/fmicb.2017.01585

Ihl, C., \& Klein, D. R. (2001). Habitat and Diet Selection by Muskoxen and Reindeer in Western Alaska. The Journal of Wildlife Management , 65 (4), 964-972. https://doi.org/10.2307/3803045

Kinross, J. M., Darzi, A. W., \& Nicholson, J. K. (2011). Gut microbiome-host interactions in health and disease. Genome Medicine , 3 (3), 1-12. https://doi.org/10.1186/gm228

Klindworth, A., Pruesse, E., Schweer, T., Peplies, J., Quast, C., Horn, M., \& Glöckner, F. O. (2013). Evaluation of general $16 \mathrm{~S}$ ribosomal RNA gene PCR primers for classical and next-generation sequencingbased diversity studies. Nucleic Acids Research , 41 (1), e1. https://doi.org/10.1093/nar/gks808 
Kohl, K. D., Weiss, R. B., Cox, J., Dale, C., \& Dearing, M. D. (2014). Gut microbes of mammalian herbivores facilitate intake of plant toxins.Ecology Letters , 17 (10), 1238-1246. https://doi.org/10.1111/ele.12329

Kottek, M., Grieser, J., Beck, C., Rudolf, B., \& Rubel, F. (2006). World Map of the Köppen-Geiger climate classification updated.Meteorologische Zeitschrift , 15 , 259-263. https://doi.org/10.1127/0941$2948 / 2006 / 0130$

Martin, M. (2011). Cutadapt removes adapter sequences from high-throughput sequencing reads. EMBnet. Journal , 17 (1), 10-12. https://doi.org/10.14806/ej.17.1.200

Meehan, C. J., \& Beiko, R. G. (2014). A phylogenomic view of ecological specialization in the lachnospiraceae, a family of digestive tract-associated bacteria. Genome Biology and Evolution ,6 (3), 703-713. https://doi.org/10.1093/gbe/evu050

Morrison, D. J., \& Preston, T. (2016). Formation of short chain fatty acids by the gut microbiota and their impact on human metabolism. Gut Microbes , 7 (3), 189-200. https://doi.org/10.1080/19490976.2015.1134082

Moeller, A. H., Suzuki, T. A., Phifer-Rixey, M., \& Nachman, M. W. (2018) Transmissino modes of the mammalian gut microbiota. Science, 362(6413), 453-457. https://doi.org/10.1126/science.aat7164

Mosbacher, J. B., Michelsen, A., Stelvig, M., Hendrichsen, D. K., \& Schmidt, N. M. (2016). Show me your rump hair and i will tell you what you ate - The dietary history of muskoxen (Ovibos moschatus ) revealed by sequential stable isotope analysis of guard hairs.PLoS ONE , 11 (4), e0152874. https://doi.org/10.1371/journal.pone.0152874

Parnell, A. (2019). Simmr: A Stable Isotope Mixing Model. R package version 0.4.1. CRAN. R-Project . https://doi.org/10.1002/env.2221>.Depends

Salgado-Flores, A., Bockwoldt, M., Hagen, L. H., Pope, P. B., \& Sundset, M. A. (2016). First insight into the faecal microbiota of the high arctic muskoxen (Ovibos moschatus ). Microbial Genomics , 2 (7), e000066. https://doi.org/10.1099/mgen.0.000066

Schmidt, N. M., Grøndahl, C., Evans, A. L., Desforges, J. P., Blake, J., Hansen, L. H., Beumer, L. T., Mosbacher, J. B., Stelvig, M., Greunz, E. M., Chimienti, M., \& van Beest, F. M. (2020). On the interplay between hypothermia and reproduction in a high arctic ungulate. Scientific Reports , 10 (1), 1-7. https://doi.org/10.1038/s41598-020-58298-8

Staaland, H., \& Olesen, C. R. (1992). Muskox and caribou adaptation to grazing on the Angujaartorfiup Nunaa range in West Greenland.Rangifer , 12 (3), 105-113. https://doi.org/10.7557/2.12.2.1027

Sundset, M. A., Præsteng, K. E., Cann, I. K. O., Mathiesen, S. D., \& MacKie, R. I. (2007). Novel rumen bacterial diversity in two geographically separated sub-species of reindeer. Microbial Ecology , 54 (3), 424438. https://doi.org/10.1007/s00248-007-9254-x

Swan, G. J. F., Bearhop, S., Redpath, S. M., Silk, M. J., Goodwin, C. E. D., Inger, R., \& McDonald, R. A. (2020). Evaluating Bayesian stable isotope mixing models of wild animal diet and the effects of trophic discrimination factors and informative priors. Methods in Ecology and Evolution , 11 (1), 139-149. https://doi.org/10.1111/2041-210X.13311

Thing, H., Klein, D. R., Jingfors, K., \& Holt, S. (1987). Ecology of muskoxen in Jameson Land, northeast Greenland. Ecography ,10 (2), 95-103. https://doi.org/10.1111/j.1600-0587.1987.tb00744.x

Vacca, M., Celano, G., Calabrese, F. M., Portincasa, P., Gobbetti, M., \& de Angelis, M. (2020). The controversial role of human gut Lachnospiraceae. Microorganisms , 8 (4), 573. https://doi.org/10.3390/microorganisms 8040573

Wang, C., Dong, D., Wang, H., Müller, K., Qin, Y., Wang, H., \& Wu, W. (2016). Metagenomic analysis of microbial consortia enriched from compost: New insights into the role of Actinobacteria in lignocellulose decomposition. Biotechnology for Biofuels , 9 (1), 1-17. https://doi.org/10.1186/s13068-016-0440-2 
Wang, S., Ryan, C. A., Boyaval, P., Dempsey, E. M., Ross, R. P., \& Stanton, C. (2020). Maternal vertical transmission affecting early-life microbiota development, Trends in Microbiology, 28(1), 28-45. https://doi.org/10.1016/j.tim.2019.07.010

Xu, Z., \& Knight, R. (2015). Dietary effects on human gut microbiome diversity. British Journal of Nutrition , 113 (S1), S1-S5. https://doi.org/10.1017/S0007114514004127

Yoon, S.-H., Ha, S.-M., Kwon, S., Lim, J., Kim, Y., Seo, H., \& Chun, J. (2017). Introducing EzBioCloud: A taxonomically united database of $16 \mathrm{~S}$ rRNA gene sequences and whole-genome assemblies. International Journal of Systematic and Evolutionary Microbiology , 67 (5), 1613-1617. https://doi.org/10.1099/ijsem.0.001755

Zielińska, S., Kidawa, D., Stempniewicz, L., Łos, M., \& Łos, J. M. (2016). New insights into the microbiota of the Svalbard ReindeerRangifer tarandus platyrhynchus . Frontiers in Microbiology , 7 , 170. https://doi.org/10.3389/fmicb.2016.00170

Table 1. Previous and this studies on gut microbiome proportion of close large herbivorous

\begin{tabular}{|c|c|c|c|}
\hline Host species & $\begin{array}{l}\text { Average percentage } \\
\text { of dominant phyla }\end{array}$ & $\begin{array}{l}\text { Average percentage } \\
\text { of dominant families }\end{array}$ & References \\
\hline Muskox (adult) & $\begin{array}{l}\text { Firmicutes }(94.36 \%) \\
\text { Verrucomicrobia }(1.77 \%)\end{array}$ & $\begin{array}{l}\text { Ruminococcaceae } \\
(73.90 \%) \\
\text { Christensenellaceae } \\
(8.28 \%) \text { Lachnospiraceae } \\
(8.27 \%)\end{array}$ & this study \\
\hline Muskox (calf) & $\begin{array}{l}\text { Firmicutes }(94.03 \%) \\
\text { Verrucomicrobia }(3.31 \%)\end{array}$ & $\begin{array}{l}\text { Ruminococcaceae } \\
(56.25 \%) \\
\text { Lachnospiraceae }(24 \%)\end{array}$ & \\
\hline Muskox & $\begin{array}{l}\text { Firmicutes }(73.8 \%) \\
\text { Bacteroidetes }(23.4 \%)\end{array}$ & $\begin{array}{l}\text { Ruminococcaceae } \\
(47.1 \%) \text { Lachnospiraceae } \\
(16.5 \%)\end{array}$ & $\begin{array}{l}\text { Salgado-Flores et al., } \\
2016\end{array}$ \\
\hline Muskox & $\begin{array}{l}\text { Firmicutes }(83 \%) \\
\text { Bacteroidetes }(7 \%)\end{array}$ & $\begin{array}{l}\text { Ruminococcaceae }(66 \%) \\
\text { Lachnospiraceae }(13 \%)\end{array}$ & Bird. 2019 \\
\hline Svalbard reindeer & $\begin{array}{l}\text { Firmicutes }(56.53 \%) \\
\text { Bacteroidetes }(39.17 \%)\end{array}$ & $\begin{array}{l}\text { Ruminococcaceae } \\
(35.17 \%) \text { Bacteroidaceae } \\
(34.30 \%)\end{array}$ & Zielińska et al., 2016 \\
\hline $\begin{array}{l}\text { Norwegian reindeer } \\
\text { (semi-domesticated) }\end{array}$ & $\begin{array}{l}\text { Firmicutes }(70.6 \%) \\
\text { Bacteroidetes }(29.4 \%)\end{array}$ & & Sundset et al., 2007 \\
\hline
\end{tabular}

* Dominant phyla without percentage were ordered by abundance.

\section{Data Accessibility Statement}

The data that support the findings of this study are openly available in NCBI Sequence Read Archive (SRA) database under accession number 'PRJNA753257' (https://www.ncbi.nlm.nih.gov/sra/?term=PRJNA753257)

\section{Competing Interests Statement}

The authors have declared that no competing interests exist.

\section{Acknowledgements}

We thank help from Danish-Korean expedition team during the field seasons. The research has been conducted under permissions from the Greenland government and these permits include consideration and approval of fecal sampling for muskoxen (permission no. G16-074, C-17-4). The local ethics committee 
(Ministry of Industry and Mineral Resources, the Government of Greenland) specifically reviewed and approved the application for survey license on the use of biological resources for commercial and research purposes (in the following: Act on Biological Resources).

\section{Author Contributions}

Ji-Yeon Cheon: Formal Analysis (equal); Writing-original draft (lead); Writing-review \& editing (equal): Hyunjun Cho: Formal Analysis (equal); Data curation (equal), Writing-review \& editing (equal); Mincheol Kim: Formal Analysis (equal); Data curation (equal), Writing-review \& editing (equal); Hyun Je Park: Formal Analysis (equal); Writing-review \& editing (equal); Tae-Yoon S. Park: Supervision (equal); Funding acquisition (lead); Writing-review \& editing (equal); Won Young Lee: Conceptualization (lead); Investigation (lead); Formal Analysis (equal); Supervision (equal), Writing-original draft (lead), Writing - Review \& Editing (lead)

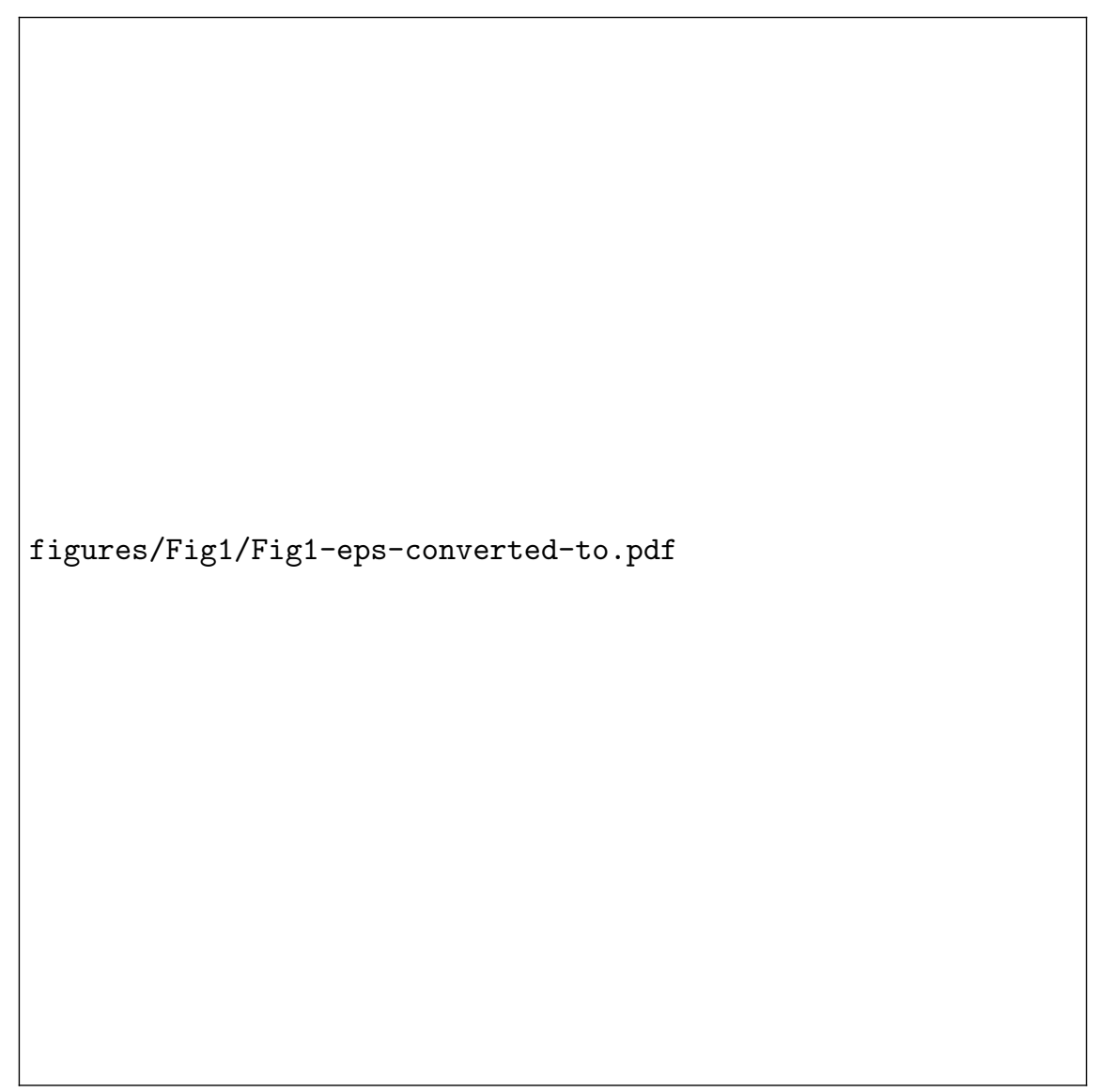


figures/Fig2/Fig2-eps-converted-to.pdf 
figures/Fig3/Fig3-eps-converted-to.pdf 
figures/Fig4/Fig4-eps-converted-to.pdf 
figures/Fig5/Fig5-eps-converted-to.pdf 
figures/Fig6/Fig6-eps-converted-to.pdf 
figures/Fig7/Fig7-eps-converted-to.pdf 
figures/Fig8/Fig8-eps-converted-to.pdf 
figures/Fig9/Fig9-eps-converted-to.pdf 
figures/Fig10/Fig10-eps-converted-to.pdf 